\title{
La obesidad incrementa la probabilidad de presentar pólipos adenomatosos colorrectales
}

\author{
Cristián Díaz-Solleiro ${ }^{1 *}$, Estefanía E. de la Cruz-Castillo², Hugo López-Acevedo ${ }^{1}$ y \\ Carlos J. Mata-Quintero ${ }^{1}$ \\ ${ }^{1}$ Departamento de Endoscopia Gastrointestinal, Hospital Central Norte de Petróleos Mexicanos, Ciudad de México; ${ }^{2}$ Facultad de Ciencias de la \\ Salud, Universidad Anáhuac México Norte, Huixquilucan, Estado de México. México
}

\section{Resumen}

Introducción: En estudios recientes se ha encontrado una relación entre obesidad y pólipos colorrectales. En México, un porcentaje importante de la población sufre de obesidad, la cual no se considera como factor de riesgo independiente para el tamizaje de cáncer colorrectal. Objetivos: Determinar el riesgo que tienen los pacientes obesos de presentar pólipos adenomatosos colorrectales en comparación con los no obesos, ya que representan la lesión precursora del cáncer de colon. Materiales y métodos: Se realizó un estudio de tipo transversal, retrospectivo, con pacientes sometidos a colonoscopia en los hospitales centrales de Petróleos Mexicanos en un periodo de cuatro años. Se revisaron un total de 614 expedientes y las variables que se estudiaron fueron: edad, sexo, índice de masa corporal, antecedentes personales y familiares de cáncer de colon, colitis ulcerativa de más de 10 años y diagnóstico histopatológico. Con el fin de determinar el impacto de la obesidad en la aparición de pólipos adenomatosos se llevó a cabo una regresión lineal múltiple para evaluar en qué medida se relacionaban ambas variables, y se calculó la razón de momios para determinar el objetivo principal del estudio. Resultados: Se incluyeron 614 pacientes, el 69.4\% eran mujeres y la edad promedio de $60.91 \pm 14.66$ años. La prevalencia de pólipos adenomatosos es del 17.4\%. Destacan el sobrepeso y la obesidad, con una frecuencia de 242 (39.4\%) y 117 casos (19.1\%) respectivamente. El análisis de regresión encontró una relación entre obesidad y presencia de pólipos adenomatosos del 3.6\%, y se calculó una razón de momios de 2.8. Conclusiones: En la población estudiada se encontró una probabilidad de casi tres veces más para presentar pólipos adenomatosos colorrectales en personas obesas en comparación con aquellas que no lo son.

Palabras clave: Obesidad. Pólipos. Colon. Cáncer.

\section{Obesity increases the probability of presenting colorectal adenomatous polyps}

\section{Abstract}

Introduction: In recent studies a relationship between obesity and colorectal polyps has been found. In Mexico, a significant percentage of the population suffers from obesity, which isn't considered an independent risk factor for colorectal cancer screening. Objectives: Determine whether obese patients have colorectal adenomatous polyps more frequently in comparison with non-obese patients, as these lesions represent the precursor of colon cancer. Materials and methods: A cross-sectional, retrospective study was carried out, with patients undergoing colonoscopy 
in the central hospitals of Petróleos Mexicanos over a period of 4 years. We reviewed a total of 614 records and the variables studied were: age, sex, body mass index, personal and family history of colon cancer, ulcerative colitis over 10 years and histopathological diagnosis. To determine the impact of obesity on the appearance of adenomatous polyps, we perform a multiple linear regression technique to evaluate the extent to which the variables are related, and the odds ratio has been calculated to determine the main objective of the study. Results: 614 patients were included, $69.4 \%$ were women, and the average age is $60.91+14.66$ years. The prevalence of adenomatous polyps is $17.4 \%$. Overweight and obesity stand out with a frequency of $242(39.4 \%)$ and 117 cases (19.1\%) respectively. The analysis of the relationship between obesity and the presence of adenomatous polyps was $3.6 \%$, with an odds ratio of 2.8. Conclusions: In the studied population, obese patients had colorectal adenomatous polyps almost 3 times more frequently compared to those who are not.

Keywords: Obesity. Polyps. Colon. Cancer.

\section{Introducción}

El cáncer de colon es la tercera causa mundial de cáncer ${ }^{1}$ y la cuarta en México, tanto en mujeres como en hombres, responsable de 700,000 muertes al año². En la última década, ha habido un incremento del $36 \%$ en la incidencia; además, la edad de presentación cada vez es más temprana ${ }^{3,4}$.

Dentro de los principales factores de riesgo se incluyen la enfermedad inflamatoria intestinal, antecedentes heredofamiliares de cáncer colorrectal (AHFCCR), ingesta de carne roja, tabaquismo, sedentarismo, bajo consumo de frutas y vegetales ${ }^{5-8}$, así como la obesidad $^{9-15}$. En cuanto al tipo histopatológico, el adenocarcinoma es el más frecuente y representa el $95 \%$ de los casos, siendo los pólipos adenomatosos los precursores de esta variante, cuya detección es la base de las guías para el manejo oportuno de estas lesiones ${ }^{16-20}$.

En México, alrededor del $70 \%$ de la población adulta sufre de sobrepeso y obesidad ${ }^{21-23}$. Actualmente no se considera la obesidad como factor de riesgo independiente para el tamizaje de cáncer de colon y pólipos adenomatosos. Las guías para el tamizaje de cáncer de colon en pacientes con riesgo promedio recomiendan que este se inicie a partir de los 50 años con colonoscopia cada 10 años más prueba inmunoquímica fecal anual (FIT), como segunda opción colonografía por tomografía cada cinco años más FIT cada tres años más sigmoidoscopia flexible cada cinco o 10 años, y como tercera opción cápsula endoscópica cada cinco años ${ }^{24-26}$.

No existen cifras de la prevalencia de obesidad ni de los casos diagnosticados de cáncer de colon y pólipos adenomatosos en esta población. La trascendencia de la identificación de estos datos y el impacto que pueda llegar a tener la obesidad sobre la aparición del cáncer de colon y pólipos adenomatosos puede promover el desarrollo de programas de detección temprana para ofrecer un tratamiento oportuno, así como una posible disminución en la frecuencia de los casos. Este precedente aportaría a las medidas que se llevan a cabo en las instituciones de salud pública, ya que no existen en la actualidad legislaciones para que se lleven a cabo pruebas de tamizaje.

\section{Material y métodos}

El presente estudio es de tipo observacional, transversal y retrospectivo. Se trabajó con pacientes que se hubiesen sometido a colonoscopia en los hospitales centrales de Petróleos Mexicanos (PEMEX) durante el periodo del 1 de enero de 2013 al 31 de diciembre de 2016, y el objetivo es determinar el riesgo que tienen los pacientes obesos de presentar pólipos adenomatosos colorrectales en comparación con pacientes no obesos en los hospitales centrales de PEMEX.

Los criterios de inclusión, exclusión y eliminación que se consideraron se describen en la tabla 1. Las variables que se tomaron en cuenta en el estudio fueron consideradas debido a su importancia como factores de riesgo para la aparición de pólipos adenomatosos reportada en la bibliografía y se describen con detalle en la tabla 2.

Para el cálculo del tamaño de muestra se utilizó el software OpenEpi v.3.01 donde se consideró la fórmula de Fleiss para estudios de tipo transversal ${ }^{27}$; considerando un nivel de significancia bilateral $(1-\alpha)$ del $95 \%$; potencia (1- $\beta$ ) del $80 \%$, razón expuesto/no expuesto de 1, porcentaje de no expuestos positivos del $30 \%$, razón de momios de 3 , estos últimos se consideraron con base en la literatura internacional y el porcentaje de expuestos positivos de 56 . Con esto se obtuvo lo mostrado en la tabla 3.

El muestreo fue de tipo probabilístico estratificado y se recolectaron los datos del expediente clínico electrónico SSS-SIAH de PEMEX; se manejaron bajo 
Tabla 1. Criterios de inclusión, exclusión y eliminación

\begin{tabular}{|c|c|c|}
\hline Criterios de inclusión & Criterios de exclusión & Criterios de eliminación \\
\hline $\begin{array}{l}\text { Pacientes que se hubiesen sometido a } \\
\text { colonoscopia en los hospitales centrales } \\
\text { de PEMEX durante el periodo del } 1 \text { de } \\
\text { enero de } 2013 \text { al } 31 \text { de diciembre de } 2016\end{array}$ & $\begin{array}{l}\text { Limpieza intestinal evaluada como mala } \\
\text { determinada por una puntuación total en la } \\
\text { escala de Boston menor a } 6\end{array}$ & Expediente incompleto \\
\hline Edad: mayores de 18 años & $\begin{array}{l}\text { Tiempo de retirada de la colonoscopia } \\
\text { menor a } 6 \text { minutos }\end{array}$ & $\begin{array}{l}\text { Expedientes duplicados: en el caso de } \\
\text { aquellos pacientes que cuenten con dos o } \\
\text { más registros de colonoscopia en el } \\
\text { periodo comprendido, se tomará en cuenta } \\
\text { únicamente el más reciente }\end{array}$ \\
\hline \multirow[t]{3}{*}{$\begin{array}{l}\text { Indicación de la colonoscopia: cualquier } \\
\text { indicación }\end{array}$} & $\begin{array}{l}\text { Colonoscopia incompleta, con observación } \\
\text { parcial de los segmentos de colon (ciego, } \\
\text { válvula ileocecal y agujero apendicular) }\end{array}$ & \\
\hline & Reporte endoscópico incompleto o faltante & \\
\hline & $\begin{array}{l}\text { Antecedente de pólipos colónicos y/o } \\
\text { cáncer de colon. }\end{array}$ & \\
\hline
\end{tabular}

PEMEX: Petróleos Mexicanos.

Tabla 2. Variables

\begin{tabular}{|c|c|c|c|c|}
\hline Variable & Definición conceptual & Definición operacional & Indicador & Escala de medición \\
\hline Edad & $\begin{array}{l}\text { Tiempo transcurrido a } \\
\text { partir del nacimiento de } \\
\text { un individuo }\end{array}$ & $\begin{array}{l}\text { Años de vida transcurridos al } \\
\text { momento del estudio }\end{array}$ & Años & Razón discreta \\
\hline Sexo & $\begin{array}{l}\text { Identidad sexual de una } \\
\text { persona }\end{array}$ & $\begin{array}{l}\text { Determinación del sexo } \\
\text { biológico del paciente }\end{array}$ & $\begin{array}{l}\text { Hombre } \\
\text { Mujer }\end{array}$ & Nominal \\
\hline IMC & $\begin{array}{l}\text { Es una razón matemática } \\
\text { que asocia la masa y la } \\
\text { talla de un individuo }\end{array}$ & $\begin{array}{l}\text { Relación de la talla y el peso } \\
\text { del individuo encontrada en el } \\
\text { expediente clínico y calculada por } \\
\text { la siguiente fórmula: IMC = peso/ } \\
\text { (talla)2. Estratificado de acuerdo } \\
\text { con los criterios de la OMS }\end{array}$ & $\begin{array}{l}\text { Bajo peso } \\
\text { Normal } \\
\text { Sobrepeso } \\
\text { Obesidad grado I, } \\
\text { obesidad grado II y } \\
\text { obesidad grado III }\end{array}$ & Ordinal \\
\hline $\begin{array}{l}\text { Antecedentes } \\
\text { personales } \\
\text { patológicos de } \\
\text { cáncer en cualquier } \\
\text { otro sitio distinto al } \\
\text { colon y/o recto }\end{array}$ & $\begin{array}{l}\text { Registro de antecedente } \\
\text { de cáncer del paciente } \\
\text { en cualquier sitio distinto } \\
\text { al colon y/o recto. }\end{array}$ & $\begin{array}{l}\text { Se tomará en cuenta el } \\
\text { registrado en el expediente } \\
\text { clínico en el apartado de } \\
\text { antecedentes personales } \\
\text { patológicos. }\end{array}$ & $\begin{array}{l}\text { Sí } \\
\text { No }\end{array}$ & Nominal \\
\hline AHFCCR & $\begin{array}{l}\text { Registro de las relaciones } \\
\text { entre los miembros de } \\
\text { una familia junto con sus } \\
\text { antecedentes médicos de } \\
\text { cáncer de colon y/o recto }\end{array}$ & $\begin{array}{l}\text { Se tomará en cuenta el registrado } \\
\text { en el expediente clínico en el } \\
\text { apartado de antecedentes } \\
\text { heredofamiliares, siempre y } \\
\text { cuando este antecedente sea de } \\
\text { familiar de primer grado }\end{array}$ & $\begin{array}{l}\text { Sí } \\
\text { No }\end{array}$ & Nominal \\
\hline $\begin{array}{l}\text { CUCI de más de } 10 \\
\text { años de evolución }\end{array}$ & $\begin{array}{l}\text { Diagnóstico clínico e } \\
\text { histopatológico de colitis } \\
\text { ulcerativa mayor o igual } \\
\text { a } 10 \text { años }\end{array}$ & $\begin{array}{l}\text { Se tomará en cuenta el } \\
\text { diagnóstico registrado en el } \\
\text { expediente clínico del paciente }\end{array}$ & $\begin{array}{l}\text { Sí } \\
\text { No }\end{array}$ & Nominal \\
\hline $\begin{array}{l}\text { Diagnóstico } \\
\text { histopatológico }\end{array}$ & $\begin{array}{l}\text { Determinación del tipo } \\
\text { histopatológico mediante } \\
\text { los hallazgos } \\
\text { microscópicos en el } \\
\text { contexto de la historia del } \\
\text { paciente }\end{array}$ & $\begin{array}{l}\text { Se tomará en cuenta el } \\
\text { diagnóstico histopatológico de } \\
\text { la lesión registrado en el } \\
\text { expediente clínico del paciente } \\
\text { correspondiente a la toma de } \\
\text { biopsia durante la colonoscopia }\end{array}$ & $\begin{array}{l}\text { Adenomatosos: } \\
\text { tubulares, vellosos, } \\
\text { tubulovellosos, no } \\
\text { especificado } \\
\text { Hiperplásicos o } \\
\text { serrados } \\
\text { Misceláneos: } \\
\text { hamartomatosos, } \\
\text { inflamatorios }\end{array}$ & Nominal \\
\hline
\end{tabular}


Tabla 3. Tamaño de la muestra*

\begin{tabular}{l|c|c|}
\hline Tamaño de la muestra & $\mathbf{n}$ & $\mathbf{n + 2 0 \%}$ de pérdidas \\
\hline Expuestos & 55 & 66 \\
\hline No expuestos & 55 & 66 \\
\hline Total del tamaño de la muestra & 110 & 132 \\
\hline *Se especifica el número de pacientes requeridos como mínimo para establecer \\
un nivel de significancia bilateral del 95\%, potencia del 80\% y pérdida del 20\%.
\end{tabular}

Tabla 4. Demarcación pronóstica de la muestra*

\begin{tabular}{|l|c|}
\hline Características & $\mathbf{n}=614(\%)$ \\
\hline Sexo femenino & $426(69.4)$ \\
\hline Edad (años) & $60.91 \pm 14.66$ \\
\hline IMC $\left(\mathrm{kg} / \mathrm{m}^{2}\right)$ & $27.06 \pm 4.6$ \\
\hline Presencia de pólipos & $156(25.4)$ \\
\hline $\begin{array}{l}\text { Presencia de cáncer en otro sitio distinto al } \\
\text { colon y recto }\end{array}$ & $43(7)$ \\
\hline CUCl > 10 años de evolución & $8(1.3)$ \\
\hline AHFCCR & $18(2.9)$ \\
\hline
\end{tabular}

*Se reporta la media y la desviación estándar para el caso de edad e IMC, en el caso de las otras variables se reporta frecuencia absoluta y relativa. AHFCCR: antecedentes heredofamiliares de cáncer colorrectal; CUCl: colitis ulcerativa crónica idiopática; IMC: índice de masa corporal.

confidencialidad y solo para fines de este proyecto; al ser un estudio de tipo observacional, retrospectivo, no se solicitó carta de consentimiento.

Para el análisis estadístico primero se organizaron los datos en una tabla de demarcación pronóstica, reportando la media y la desviación estándar para el caso de edad e índice de masa corporal (IMC), en el caso de las otras variables se reporta frecuencia absoluta y relativa; posteriormente se realizó una categorización del IMC y del diagnóstico histopatológico, y finalmente se llevó a cabo una regresión lineal múltiple para evaluar en qué medida se relacionaba la obesidad con la aparición de pólipos adenomatosos, y se calculó la razón de momios para determinar el objetivo principal del estudio.

\section{Resultados}

Durante el periodo 2013 a 2016 se revisaron un total de 645 expedientes, de los cuales 31 se eliminaron de acuerdo con los criterios previamente señalados, con un promedio de 152.8 expedientes por año.
La muestra se integra predominantemente por pacientes de sexo femenino (69.4\%), con edades comprendidas entre los 19 y los 99 años (promedio de 60.91 años); se observó homogeneidad en el IMC, con $27.06 \mathrm{~kg} / \mathrm{m}^{2}$. Cabe mencionar que la frecuencia de pacientes con otros factores de riesgo como el diagnóstico de colitis ulcerativa crónica idiopática (CUCl) de más de 10 años y los AHFCCR es baja, siendo del 1.3 y el $2.9 \%$ respectivamente (Tabla 4).

Se determinó la prevalencia de pólipos adenomatosos colorrectales en pacientes del Servicio de Endoscopia de los hospitales centrales de PEMEX en el periodo 2013 al 2016, siendo esta del 17.4\%.

En la tabla 5 se enfatiza el IMC de la población estudiada de acuerdo con cada una de las categorías, que van desde bajo peso hasta obesidad grado III; destacan el sobrepeso y la obesidad, con una frecuencia de $242(39.4 \%)$ y 117 casos (19.1\%) respectivamente. Además, en esta tabla se reporta la presencia de pólipos en el total de la muestra y la categoría respecto a su variante histológica, siendo los más frecuentes los de tipo adenomatoso tubular (77 casos) y los hiperplásicos o serrados (46 casos); con menor frecuencia están los misceláneos, con tres casos.

Con el objetivo de determinar el impacto de la obesidad en la aparición de pólipos adenomatosos, se llevó a cabo una regresión lineal múltiple para evaluar en qué medida se relacionaban ambas variables. Se obtuvieron los resultados que se muestran en la tabla 6.

Nótese que la variable de interés que más repercusiones tiene en la varianza explicativa (VE) es la obesidad, con un porcentaje del $3.6 \%$; mientras que el resto de las variables, como edad, AHFCCR y sexo, solo contribuyen en el $2.7 \%$; se integraron en la tabla los valores del grado de significación (p) para sobrepeso, CUCI de larga evolución y presencia de cáncer en otros sitios distintos al colon por ser relevantes al no contribuir a la VE. Debido a la importancia de estos resultados se prosiguió al análisis en particular de la obesidad en relación con la aparición de pólipos adenomatosos, calculando la razón de momios (RM) y obteniendo los valores mostrados en la figura 1.

Se determinó la chi cuadrada con un valor de Mantel-Haenszel de $22.28(p<0.001)$ y mid-p exacto como medida de asociación ( $p$ < 0.001); el cálculo de la RM fue de 2.807, cuyos límites inferior y superior corresponden a 1.801 y 4.362 respectivamente y se obtuvo una fracción etiológica en la población $\left(\frac{F E p}{R M}\right)$ del $27.1 \%$ (14.9-39.3) y la fracción etiológica en expuestos $\left(\frac{F E e}{R M}\right)$ del $64.44 \%$ (44.78-77.11). 
Tabla 5. Categorización del índice de masa corporal (IMC) y del diagnóstico histopatológico de la población

\begin{tabular}{|c|c|c|c|}
\hline & & & n (\%) \\
\hline Categoría IMC & $\begin{array}{l}\text { Bajo peso } \\
\text { Normal } \\
\text { Sobrepeso } \\
\text { Obesidad I } \\
\text { Obesidad II } \\
\text { Obesidad III }\end{array}$ & & $\begin{array}{c}8(1.3) \\
215(35) \\
242(39.4) \\
117(19.1) \\
23(3.7) \\
9(1.5)\end{array}$ \\
\hline \multirow[t]{4}{*}{$\begin{array}{l}\text { Diagnóstico histopatológico de los } \\
\text { pólipos } n=156\end{array}$} & \multicolumn{2}{|l|}{ Adenomatosos } & $\begin{array}{c}\text { Tubulares } 77(49.4) \\
\text { Vellosos } 12(7.7) \\
\text { Tubulovellosos } 17(10.9) \\
\text { No especificado } 1(0.6)\end{array}$ \\
\hline & \multicolumn{2}{|c|}{ Hiperplásicos o serrados } & $46(29.5)$ \\
\hline & Misceláneos & Hamartomatosos & $1(0.6)$ \\
\hline & & Inflamatorios & $2(1.3)$ \\
\hline
\end{tabular}

Tabla 6. Regresión lineal múltiple de la aparición de pólipos adenomatosos

\begin{tabular}{|l|c|c|c|c|}
\hline Variable & $\mathbf{r}$ & $\mathbf{r}^{2}$ & $\mathbf{V E}$ & $\boldsymbol{p}^{*}$ \\
\hline IMC $\left(\mathrm{kg} / \mathrm{m}^{2}\right)$ & 0.191 & 0.036 & $3.6 \%$ & 0.001 \\
\hline Edad (años) & 0.107 & 0.011 & $1.06 \%$ & 0.008 \\
\hline AHFCCR & 0.102 & 0.010 & $0.95 \%$ & 0.012 \\
\hline Sexo & 0.086 & 0.0074 & $0.70 \%$ & 0.033 \\
\hline $\begin{array}{l}\text { Sobrepeso } \\
\text { CUCl > 10 años de }\end{array}$ & - & - & - & 0.338 \\
\hline evolución & - & - & - & 0.113 \\
\hline $\begin{array}{l}\text { Presencia de cáncer en } \\
\text { otros sitios distintos al } \\
\text { colon y/o recto }\end{array}$ & - & - & - & 0.305 \\
\hline $\begin{array}{l}\text { Total } \\
\text { *Se considera } p<0.05 \text { como estadísticamente significativo. }\end{array}$ & & & \\
\hline $\begin{array}{l}\text { AHFCCR: antecedentes heredofamiliares de cáncer colorrectal; CUCl: colitis } \\
\text { ulcerativa crónica idionática; IMC: índice de masa corporal; VE: varianza explicativa. }\end{array}$
\end{tabular}

Para el análisis de los resultados se utilizó el paquete de análisis estadístico IBM SPSS ${ }^{\circledR}$ v.25, el GraphPad Prism ${ }^{\circledR} 2.01$ y el OpenEpi v.3.

\section{Discusión}

En la población examinada de los hospitales centrales de PEMEX, se encontró que los pacientes obesos tienen 2.8 veces más riesgo de presentar pólipos adenomatosos colorrectales en comparación con pacientes no obesos.

Es preocupante la situación del sobrepeso y la obesidad que existe en México, aunado a las comorbilidades que de ello demandan, por lo que se deben establecer medidas

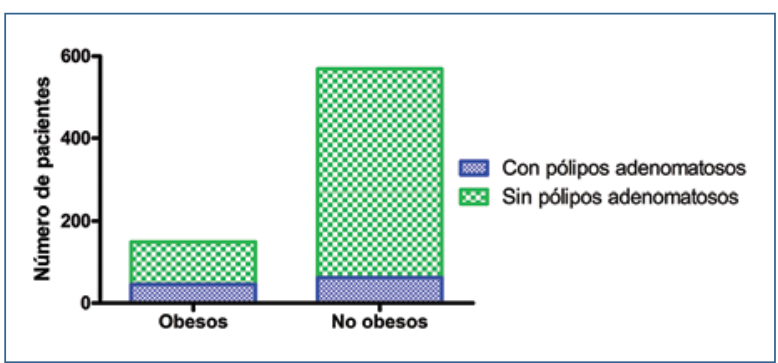

Figura 1. Se observa la distribución de pólipos adenomatosos en pacientes con y sin obesidad.

efectivas para prevenir o identificar de manera oportuna aquellas que se pueden evitar, como el cáncer de colon y recto.

La identificación de pólipos, como los pólipos adenomatosos (que representan una gran amenaza), es fundamental para dar un tratamiento temprano; por lo que la causa de consulta por primera vez no debería desembocar en un «hallazgo incidental».

La necesidad que existe en México es real, ya que la población mayormente expuesta a factores de riesgo modificables representa más del $60 \%$, presentando un riesgo 0.5 veces mayor para padecer cáncer colorrectal.

Claramente, se deben establecer pautas nacionales para la pesquisa de esta enfermedad, que si se detectara a tiempo, tiene un buen nivel de supervivencia. Por ello, se deben sentar las bases mediante la realización de estudios como el presente.

Es alarmante que, de acuerdo con los resultados encontrados, la mayoría de la población que acude al Departamento de Endoscopia de los hospitales centrales de PEMEX padecen sobrepeso u obesidad, 
con una relación mujer: hombre de 1:0.4; estas características demográficas son de suma importancia, ya que como vimos, son factores de riesgo para cáncer colorrectal; mientras que en la población que padece obesidad, la aparición de pólipos en mujeres en relación con los hombres es de 1:1.6.

Es importante ver que se diagnosticaron pólipos adenomatosos en el $17 \%$ de la población y al $1 \%$ se le diagnosticó cáncer.

Estos hallazgos no logran dar explicación al problema, ya que solo el $6.3 \%$ de las causas están explicadas con este estudio, por lo que faltaría explicar el 93.7\% restante.

A pesar de esto, el IMC logra dar explicación al 3.6\% del fenómeno con una significancia estadística muy importante, por lo que se decidió poner mayor atención; encontrándose una RM de 2.8, lo que se traduce en una probabilidad de casi tres veces más para presentar pólipos adenomatosos colorrectales en personas obesas en comparación con aquellas personas que no lo son.

\section{Financiamiento}

La presente investigación no ha recibido ayudas específicas provenientes de agencias del sector público, sector comercial o entidades sin ánimo de lucro.

\section{Conflicto de intereses}

Los autores declaran no tener conflicto de intereses.

\section{Responsabilidades éticas}

Protección de personas y animales. Los autores declaran que para esta investigación no se han realizado experimentos en seres humanos ni en animales.

Confidencialidad de los datos. Los autores declaran que en este artículo no aparecen datos de pacientes.

Derecho a la privacidad y consentimiento informado. Los autores declaran que en este artículo no aparecen datos de pacientes.

\section{Bibliografía}

1. Bray F, Ferlay J, Soerjomataram I, Siegel RL, Torre LA, Jemal A. Global Cancer Statistics 2018: GLOBOCAN estimates of incidence and mortality worldwide for 36 cancers in 185 countries. CA Cancer J Clin 2018;68(6):394-424 .
2. Cáncer de colon y recto. Infografía desarrollada entre los institutos nacionales de Cancerología (INCan) y de Salud Pública (INSP) [Internet]. México: Instituto Nacional de Salud Pública [fecha última actualización: 4 de septiembre de 2015]. Disponible en: https://www.insp.mx/infografias/cancer-colon-recto.html

3. Burbano-Luna DF, Manrique MA, Chávez-García MA, Pérez-Corona T, Hernández-Velázquez NN, Escandón-Espinoza YM, et al. Epidemiología del cáncer colorrectal en menores de 50 años en el Hospital Juárez de México. Endoscopia. 2016;28(4):160-5.

4. Qaiyoume A, Khursheed A. Colorectal cancer in younger population: Our experience. J Pak Med Assoc. 2013;63:1275-7.

5. Cervantes-Sánchez G, Ochoa-Carrillo FJ, Ocampo-Le Royal R, et al. Cáncer de colon. GAMO. 2008;7(4):12-15.

6. Johnson IT, Lund EK. Nutrition, obesity and colorectal cancer. Aliment Pharmacol Ther. 2007;26(2):161-81.

7. Brooke $H$, Talbäck M, Martling A. Socioeconomic position and incidence of colorectal cancer in the Swedish population. Cancer Epidemiol. 2016;40:188-95.

8. Zisman AL, Nickolov A, Brand RE, Gorchow A, Roy HK. Associations between the age at diagnosis and location of colorectal cancer and the use of alcohol and tobacco. Arch Intern Med. 2006;166:629-34.

9. Im JP, Kim D, Chung SJ, Jin EH, Han YM, Park MJ, et al. Visceral obesity as a risk factor for colorectal adenoma occurrence in surveillance colonoscopy. Gastrointest Endosc. 2018;88(1):119-27.

10. Chen J, Huang XF. High fat diet-induced obesity increases the formation of colon polyps induced by azoxymethane in mice. Ann Transl Med. 2015;3(6):79.

11. Ruiz Morales OF, Otero Regino W, Gómez Zuleta MA, Castro Soteldo D. La obesidad abdominal aumenta el riesgo de pólipos colorrectales. Rev Col Gastroenterol. 2014;29(4):376-82.

12. Comstock SS, Hortos K, Kovan B, McCaskey S, Pathak DR, Fenton JI. Adipokines and obesity are associated with colorectal polyps in adult males: a cross-sectional study. PLoS One. 2014;9(1):e85939.

13. Lee JY, Kwak SM, Myung SK, Jee SH. Obesity and colorectal adenomatous polyps: a cross-sectional study in Korean adults. Obesity. 2014;22(2):518-25.

14. Bardou M, Barkun AN, Martel M. Obesity and colorectal cancer. Gut. 2013;62:933-47.

15. Ma $Y$, Yang $Y$, Wang F, Zhang $P$, Shi $C$, Zou $Y$, et al. Obesity and risk of colorectal cancer: A systematic review of prospective studies. PLoS One. 2013;8(1):e53916.

16. Arévalo F, Aragón V, Alva J, Pérez Narrea M, Cerrillo G, Montes P, et al. Pólipos colorrectales: actualización en el diagnóstico. Rev Gastroenterol Perú. 2012;32(2):123-33.

17. Castells A, Bessa X. Pólipos y poliposis intestinal. En: Ponce J, editor. Tratamiento de las enfermedades gastroenterológicas. Barcelona: Doyma; 2000. pp. 247-256.

18. Montgomery EA. Colon: polyps, tumors, and tumefactions. En: Montgomery EA, Voltaggio L. Biopsy interpretation of the gastrointestinal tract mucosa. Filadelfia: Lippincott Williams \& Williams; 2006. pp. 165-245.

19. Hamilton SR, Nakamura SI, Bosman FT. Carcinoma of the colon and rectum. En: WHO Classification of tumors of the digestive system. Vol. 3. Lyon: WHO Press; 2010. pp. 316-98.

20. Li S, Bugart L. Histopathology of serrated adenoma, its variants, and differentiation from conventional adenomatous and hyperplastic polyps. Arch Pathol Lab Med. 2007;131:440-5.

21. Obesity and overweight. Key Facts [Internet]. World Health Organization; 2018 [fecha de publicación: 16 de febrero de 2018]. Disponible en: http:// www.who.int/mediacentre/factsheets/fs311/en

22. Shamah T, Cuevas L. Sobrepeso y obesidad: en busca de un enfoque efectivo. México: Centro de Investigación en Nutrición y Salud, Instituto Nacional de Salud Pública; 2017.

23. Barrera-Cruz A, Rodríguez-González A, Molina-Ayala MA. Escenario actual de la obesidad en México. Rev Med Inst Mex Seguro Soc. 2013;51(3):292-9.

24. Rex DK, Boland R, Dominitz JA, Giardiello FM, Johnson DA, Kaltenbach T, et al. Colorectal cancer screening: Recommendations for physicians and patients from the U.S. Multi-Society Task Force on Colorectal Cancer. Gastrointest Endosc. 2017;86(1):18-33.

25. Guía de Práctica Clínica, Detección oportuna y diagnóstico de cáncer de colon y recto no hereditario en adultos en primero, segundo y tercer nivel de atención, México: Secretaría de Salud; 2009.

26. Provenzale D, Jasperson K, Ahnen DJ, Aslanian H, Bray T, Cannon JA, et al.; National comprehensive cancer netwok. Colorectal cancer screening, Version 1.2015. J Natl Compr Canc Netw. 2015;13:959-68.

27. Fleiss JL, Levin B, Myunghee CP. Determining sample sizes needed to detect a difference between two proportions. En: Fleiss JL, Levin B, Myunghee CP. Statistical methods for rates and proportions, Third edition. New York: John Wiley \& Sons; 2003. pp. 64-85. 